\title{
On regular factors in regular graphs with small radius
}

\author{
Arne Hoffmann \\ Lehrstuhl C für Mathematik \\ RWTH-Aachen, 52056 Aachen, Germany \\ hoffmann@mathc.rwth-aachen.de
}

\author{
Lutz Volkmann* \\ Lehrstuhl II für Mathematik \\ RWTH-Aachen, 52056 Aachen, Germany \\ volkm@math2.rwth-aachen.de
}

Submitted: Aug 21, 2001; Accepted: Nov 5, 2003; Published: Jan 2, 2004

MR Subject Classifications: 05C70, 05C35

\begin{abstract}
In this note we examine the connection between vertices of high eccentricity and the existence of $k$-factors in regular graphs. This leads to new results in the case that the radius of the graph is small $(\leq 3)$, namely that a $d$-regular graph $G$ has all $k$-factors, for $k|V(G)|$ even and $k \leq d$, if it has at most $2 d+2$ vertices of eccentricity $>3$. In particular, each regular graph $G$ of diameter $\leq 3$ has every $k$-factor, for $k|V(G)|$ even and $k \leq d$.
\end{abstract}

\section{Introduction}

All graphs considered are finite and simple. We use standard graph terminology. For vertices $u, v \in V(G)$ let $d(u, v)$ be the number of edges in a shortest path from $u$ to $v$, called the distance between $u$ and $v$. Let further $e(v):=\max \{d(v, x): x \in V(G)\}$ denote the eccentricity of $x$. The radius $r(G)$ and the diameter $\operatorname{dm}(G)$ of a graph $G$ are the minimum and maximum eccentricity, respectively. If a graph $G$ is disconnected, then $e(v):=\infty$ for all vertices $v$ in $G$.

The complete graph with $n$ vertices is denoted by $K_{n}$. For a set $S \subseteq V(G)$ let $G[S]$ be the subgraph induced by $S$. In an $r$-almost regular graph the degrees of any two vertices differ by at most $r$. For $b \geq a>0$ we call a subgraph $F$ of $G$ an $[a, b]$-factor, if $V(F)=V(G)$ and the degrees of all vertices in $F$ are between $a$ and $b$. We call a $[k, k]$-factor simply a $k$-factor. If we do not say otherwise, we quietly assume that $k<d$ if $G$ is a $d$-regular graph.

Many sufficient conditions for the existence of a $k$-factor in a regular graph are known today. Good surveys can be found in Akiyama and Kano [1] as well as Volkmann [8]. As far as we know, none of these conditions have taken the eccentricity of vertices into

\footnotetext{
${ }^{*}$ corresponding author
} 
account. It is an easy exercise to show that every regular graph $G$ with $\operatorname{dm}(G)=1$ has a $k$-factor if $k|V(G)|$ is even. For $\operatorname{dm}(G) \geq 2$ the case becomes more involved. The main result of this note is the following theorem, which provides a connection between vertices $x$ with $e(x)>3$ and the existence of a $k$-factor.

Theorem 1.1 For $d \geq 3$ let $G$ be a connected $d$-regular graph. For an integer $1 \leq k<d$ with $k|V(G)|$ even $G$ has a $k$-factor if

- $d$ and $k$ are even;

- $d$ is even, $k$ is odd and $G$ has at most $(d+1) \cdot \min \{k+1, d-k+1\}$ vertices of eccentricity $\geq 4$;

- $d$ and $k$ are odd and $G$ has at most $1+(d+2)(k+1)$ vertices of eccentricity $\geq 4$;

- $d$ is odd and $k$ is even and $G$ has at most $1+(d+2)(d-k+1)$ vertices of eccentricity $\geq 4$.

Theorem 1.1 implies the following two results as corollaries.

Theorem 1.2 A connected d-regular graph, $d \geq 2$, with at most $2 d+2$ vertices of eccentricity $\geq 4$ has every $k$-factor for $k|V(G)|$ even.

Theorem 1.3 A connected d-regular graph, $d \geq 2$, with diameter $\leq 3$ has every $k$-factor for $k|V(G)|$ even.

Theorem 1.1 is in the following way best possible: Let $d$ be even and let $k$ be odd with $d \geq 2 k+4$. Take $k+1$ copies of $K_{d+1}-u v$ and a copy of $K_{d+1}-M$, where $M$ denotes a matching of cardinality $\frac{d-2(k+1)}{2}$, as well as a vertex $x$. Connect $x$ to all vertices $u, v$ of degree $d-1$. The resulting graph $G$ is $d$-regular and has

$$
(k+1)(d-1)+2 k+3=(d+1)(k+1)+1
$$

vertices of eccentricity 4 . It further has no $k$-factor since $\Theta_{G}(\{x\}, \emptyset, k)=-2$ (see Theorem 2.1). Now let $d$ and $k$ be odd with $d \geq 3 k+6$. For an odd integer $0<p<d$ define $K_{d+2}(p):=K_{d+2}-F(p)$, where $F(p)$ denotes a [1,2]-factor such that $p$ vertices of $K^{p}$ are of degree $d-1$ and the remaining vertices are of degree $d$. Take $k+1$ copies of $K_{d+2}(3)$, one copy of $K_{d+2}(d-3(k+1))$ as well as a vertex $x$. Connect $x$ with all vertices of degree $d-1$. The resulting graph $H$ is $d$-regular and has $2+(k+1)(d+2)$ vertices of eccentricity 4. It further has no $k$-factor since $\Theta_{H}(\{x\}, \emptyset, k)=-2$.

Quite some results on factors in regular graphs have been generalized to almost regular graphs (cf. [1], [8]). Theorem 1.1, however, cannot be easily generalized to $r$-almost regular graphs:

The complete bipartite graph $K_{p, p+r}, r>0$, is $r$-almost regular and of diameter 2 but obviously has no $k$-factor. 
For complete multipartite graphs, which are $r$-almost regular and of diameter 2 , a result of Hoffman and Rodger [4] shows, that a $k$-factor only exists, if certain necessary and sufficient conditions are met.

The conditions in Theorem 1.1 are closely related to those given in the following result of Niessen and Randerath [5] on regular graphs.

Theorem 1.4 Let $n, d$ and $k$ be integers with $n>d>k \geq 1$ such that nd and nk are even. A d-regular graph of order $n$ has a $k$-factor in the following cases:

- $d$ and $k$ are even;

- $d$ is even and $k$ is odd and $n<2(d+1)$;

- $d$ and $k$ are odd and $n<1+(k+2)(d+2)$;

- $d$ is odd and $k$ is even and $n<1+(d-k+2)(d+2)$.

In all other cases there exists a d-regular graph of order $n$ without a $k$-factor.

For a regular graph with radius $\leq 3$, Theorem 1.1 provides conditions for the existence of a $k$-factor, which allow for a higher order than Theorem 1.4.

\section{Proof of the Main Theorem}

The proof of Theorem 1.1 uses the $k$-factor Theorem of Belck [2] and Tutte [7], which we cite in its version for regular graphs.

Theorem 2.1 The d-regular graph $G$ has a $k$-factor if and only if

$$
\Theta_{G}(D, S, k):=k|D|-k|S|+d|S|-e_{G}(D, S)-q_{G}(D, S, k) \geq 0
$$

for all disjoint subsets $D, S$ of $V(G)$. Here $q_{G}(D, S, k)$ denotes the number of components $C$ of $G-(D \cup S)$ satisfying

$$
e_{G}(S, V(C))+k|V(C)| \equiv 1 \quad(\bmod 2) .
$$

We simply call these components odd.

It always holds $\Theta_{G}(D, S, k) \equiv k|V(G)| \quad(\bmod 2)$ for all disjoint subsets $D, S$ of $V(G)$, whether $G$ has a $k$-factor or not.

In 1985, Enomoto, Jackson, Katerinis and Saito [3] proved the following result.

Lemma 2.2 Let $G$ be a graph and $k$ a positive integer with $k|V(G)|$ even. If $D, S \subset$ $V(G)$ such that $\Theta_{G}(D, S, k) \leq-2$ with $|S|$ minimum over all such pairs, then $S=\emptyset$ or $\Delta(G[S]) \leq k-2$. 
For regular graphs without a $k$-factor, for odd $k$, we can give the following result on the subsets $D$ and $S$.

Lemma 2.3 Let $n, k, d$ be integers such that $n$ is even and $k$ is odd with $n>d>k>0$. Let further $2 k \leq d$ if $d$ is even. If a connected d-regular graph $G$ of order $n$ has no $k$-factor, then for all disjoint subsets $D, S$ of $V(G)$ with $\Theta_{G}(D, S, k) \leq-2$ it holds $|D|>|S|$.

Proof. If $G$ does not have a $k$-factor, then, since $k n$ is even, there exist disjoint subsets $D, S$ of $V(G)$ with $\Theta_{G}(D, S, k) \leq-2$. Since $G$ is connected, $D \cup S \neq \emptyset$. Let $q:=q_{G}(D, S, k)$ and $W:=G-(D \cup S)$.

Case 1: Let $\mathbf{d}$ be even. The graph $G$ is connected and of even degree $d$, thus at least 2-edge-connected, and we get

$$
e_{G}(D \cup S, V(W)) \geq 2 q .
$$

Since $e_{G}(D, S) \leq \min \left\{d|D|-e_{G}(D, V(W)), d|S|-e_{G}(S, V(W))\right\}$, we have

$$
2 e_{G}(D, S) \leq d(|D|+|S|)-e_{G}(D \cup S, V(W)),
$$

which together with (2) results in $2 q \leq d(|D|+|S|)-2 e_{G}(D, S)$. Taking (1) into account leads to $(d-2 k)(|D|-|S|) \geq 4$, giving us the desired result.

Case 2: Let $\mathbf{d}$ be odd. We get for every odd component $C$ of $W$

$$
\begin{aligned}
e_{G}(D, V(C)) & =d|V(C)|-e_{G}(S, V(C))-2|E(C)| \\
& \equiv k|V(C)|+e_{G}(S, V(C))-2|E(C)| \equiv 1 \quad(\bmod 2)
\end{aligned}
$$

Thus $e_{G}(D, S) \leq d|D|-q$ which gives us in (1)

$$
k(|D|-|S|)+d|S|-q+2 \leq e_{G}(D, S) \leq d|D|-q,
$$

leading to

$$
(d-k)(|D|-|S|) \geq 2
$$

Proof of Theorem 1.1. The first case follows from the well-known Theorem of Petersen [6].

In the remaining cases let, without loss of generality, $k$ be odd and furthermore $2 k \leq d$ if $d$ is even, as the graph $G$ has a $k$-factor if and only if $G$ has a $(d-k)$-factor. We are only going to prove the case that $d$ and $k$ are both odd. The proof to the case $d$ even and $k$ odd only differs in the number of vertices of eccentricity $\geq 4$ and uses analogous argumentation.

Assume that $G$ does not have a $k$-factor. With Theorem 2.1 there exist disjoint subsets $D, S$ of $V(G)$ such that $\Theta_{G}(D, S, k) \leq-2$. From Lemma 2.3 we know that $|D|>|S|$ and $q \geq k(|D|-|S|)+2 \geq k+2$. 
Let $X:=\{v \in V(G): e(v) \geq 4\}$ and $C^{X}:=V(C) \cap X$ for every odd component $C$ of $W$. By the hypothesis we have $r:=|X| \leq 1+(d+2)(k+1)$. Call an odd component $C$ an $A$-component, if $|C| \leq d$ and let $a$ denote the number of $A$-components. For every $A$-component $C$ it holds $e_{G}(D \cup S, V(C)) \geq d$.

Case 1: There exist at most two odd components which have a vertex $x$ such that $e_{G}(x, D \cup S)=0$. Let $l, 0 \leq l \leq 2$, be the number of such odd components of $W$. Then these are not $A$-components, giving us $a \leq q-l$, and it holds $e_{G}(V(C), D \cup S) \geq|V(C)|$ for all other odd components. This results in

$$
\begin{aligned}
e_{G}(V(W), D \cup S) & \geq a d+(q-a-l)(d+1)+l \\
& =q(d+1)-a-l d \\
& \geq q(d+1)-(q-l)-l d \\
& =d(q-l)+l>d(q-2) .
\end{aligned}
$$

This together with (3) results in

$$
d(|D|+|S|)-2 e_{G}(D, S)>d(q-2) .
$$

Inequality (4) and $\Theta_{G}(D, S, k) \leq-2$ lead to

$$
(d-2 k)(|D|-|S|)>(d-2) q-2 d+4 .
$$

If we now use $q \geq 2+k(|D|-|S|)$, we get

$$
(d-2 k)(|D|-|S|)>(d-2)(2+k(|D|-|S|))-2 d+4,
$$

giving us the contradiction

$$
0 \geq d(1-k)(|D|-|S|)>2(d-2)+4-2 d=0 .
$$

Case 2: There exist at least three odd components having a vertex $x$ such that $e_{G}(x, D \cup S)=0$. Assume that one of these vertices is not a member of $X$. Then $e(x) \leq 3$ for this vertex and we have $e_{G}(V(C), D \cup S) \geq|V(C)|$ for all other odd components. Analogously to $l=1$ in Case 1 we can then show $e_{G}(V(W), D \cup S)>(q-2) d$ and arrive at the contradiction (5). Thus each vertex $x$ with $e_{G}(x, D \cup S)=0$ is a member of $X$. Let $\mathcal{B}$ denote the set of all odd components of $W$ which are not $A$-components. Then $|\mathcal{B}| \geq 3$ and $a \leq q-3$ and it holds

$$
\begin{aligned}
e_{G}(V(W), D \cup S) & \geq a d+\sum_{C \in \mathcal{B}}\left(|V(C)|-\left|C^{X}\right|\right) \\
& \geq a d-r+\sum_{C \in \mathcal{B}}|V(C)| \\
& \geq a d-r+(q-a)(d+1) \\
& =q(d+1)-a-r .
\end{aligned}
$$


This combined with $(3)$ and $\Theta_{G}(D, S, k) \leq-2$ leads to

$$
(d-2 k)(|D|-|S|) \geq q(d-1)+4-a-r .
$$

Since $a \leq q-3, q \geq k(|D|-|S|)+2$ and $r \leq 1+(d+2)(k+1)$, we can deduce the inequality

$$
d(1-k)(|D|-|S|) \geq 2 d+2-(d+2)(k+1),
$$

which does not give us any information in the case $k=1$. Let us first consider $k \geq 3$. Then inequality (7) can be rewritten as

$$
|D|-|S| \leq \frac{(d+1)(k+1)-2 d-3}{d(k-1)}=1+\frac{k-2}{d(k-1)}<2 .
$$

By Lemma 2.3 it follows that $|D|=|S|+1$. Let now $q=k+2+\eta$ with a non-negative integer $\eta$. With (6) and $|D|=|S|+1$ we get

$$
\begin{aligned}
a & \geq(k+2+\eta)(d-1)-d+2 k+4-1-(d+2)(k+1) \\
& =\eta(d-1)-k-1
\end{aligned}
$$

Since $q \geq a+3$ we get $k+\eta-1 \geq \eta(d-1)-k-1$, or $2 k \geq \eta(d-2)$. Thus $\eta \leq 2$ with equality if and only if $k=d-2$. Since $q \leq k+4$, the inequality $\Theta_{G}(D, S, k) \leq-2$ yields $d|S|-e_{G}(D, S) \leq 2$ and thus $e_{G}(V(W), D \cup S) \leq d+2$. For $a \geq 1$ there are at most 2 edges leading to non- $A$-components, which together with $q \geq a+3$ and the connectivity of $G$ yields a contradiction.

For $\eta \geq 1$, we have $a \geq 1$, so it remains the case $\eta=0$ and $a=0$, giving us $|S|=0$ or $e_{G}(D, S)=d|S|$ and hence $e_{G}(V(W), D) \leq d$. Since $a=0$ and from the definition of the odd components in Theorem 2.1, every odd component of $G-(D \cup S)$ has at least $d+2$ vertices. Thus $W$ has at least $(k+2)(d+2)$ vertices, of whom at most $r \leq 1+(d+2)(k+1)$ are not connected to $D$ with an edge. This means

$$
e_{G}(V(W), D) \geq(k+2)(d+2)-1-(d+2)(k+1)=d+1,
$$

which yields a contradiction.

It remains the case that $k=1$. According to Lemma 2.2, we have $|S|=0$, if we take $D$ and $S$ such that $S$ is of minimum order. Thus $q \geq|D|+2$. From the definition of odd components we have $|V(C)| \geq d+2$ for every non-A-component $C$. This gives us

$$
\begin{aligned}
e_{G}(V(W), D) & \geq a d+(q-a)(d+2)-r \\
& \geq q(d+2)-2 a-1-2(d+2) \\
& \geq q d-2 d+1 \\
& \geq(|D|+2) d-2 d+1 \\
& \geq d|D|+1,
\end{aligned}
$$

which contradicts $e_{G}(V(W), D) \leq d|D|$. 


\section{References}

[1] J. Akiyama and M. Kano, Factors and factorizations of graphs - a survey, J. Graph Theory 9 (1985) 1-42.

[2] H.B. Belck, Reguläre Faktoren von Graphen, J. Reine Angew. Math. 188 (1950) $228-252$.

[3] H. Enomoto, B. Jackson, P. Katerinis and A. Saito, Toughness and the existence of $k$-factors, J. Graph Theory 9 (1985) 87-95.

[4] D.G. Hoffman, C.A. Rodger, On the number of edge-disjoint one factors and the existence of $k$-factors in complete multipartite graphs, Discrete Math. 160 (1996) $177-187$.

[5] T. Niessen and B. Randerath, Regular factors of simple regular graphs and factorspectra, Discrete Math. 185 (1998) 89-103.

[6] J. Petersen, Die Theorie der regulären graphs, Acta Math. 15 (1891) 193-220.

[7] W.T. Tutte, The factors of graphs, Canad. J. Math. 4 (1952) 314-328.

[8] L. Volkmann, Regular graphs, regular factors, and the impact of Petersen's Theorems, Jahresber. Deutsch. Math.-Verein. 97 (1995) 19-42. 